\title{
ROLES DE GÉNERO Y DIVERSIDAD, VALIDACIÓN DE UNA ESCALAEN DOCENTES UNIVERSITARIOS DEL SUROCCIDENTE COLOMBIANO.
}

\section{GENDER ROLES AND DIVERSITY, VALIDATION OF A SCALE IN UNIVERSITY TEACHERS FROM COLOMBIAN'S SOUTHWESTERN}

Zambrano Guerrero Christian Alexander ${ }^{1}$

López Narváez Carlos Daniel²

Muñoz Muñoz Diego Fernando³.

Universidad de Nariño

\section{RESUMEN}

El estudio tuvo como objetivo analizar las propiedades psicométricas de la adaptación

$\overline{1}$ Magister en educación desde la diversidad, Doctorando en educación. Docente hora cátedra, Universidad de Nariño. Número telefónico: 3173268085. Dirección electrónica: cazambranopsi@gmail.com Universidad de Nariño, Departamento de Nariño, Colombia.

2 Egresado, Departamento de Psicología. Número telefónico: 3218356119. Dirección electrónica: lopezdaniel@ udenar.edu.co Universidad de Nariño, Departamento de Nariño, Colombia.

3 Egresado, Departamento de Psicología. Número telefónico: 3136796460. Dirección electrónica: dife354@ gmail.com Universidad de Nariño, Departamento de Nariño, Colombia. AUTOR PARA CORRESPONDENCIA. cultural del instrumento "escala de roles de género" en docentes universitarios. Método: Estudio cuantitativo de carácter descriptivo e instrumental que contó con la participaron 232 docentes de una universidad de Colombia. Se realizaron análisis de consistencia interna y de validez de los factores de la escala. Resultados: En el procedimiento de adaptación, los ajustes realizados se consideraron pertinentes tanto en el análisis de jueces como en el pilotaje. La estructura factorial de tres factores explicó el $64,677 \%$ de la varianza, aunque se evidenciaron cambios en la ubicación de tres ítems, lo cual 
lleva a la discusión teórica de tales factores. La escala y sus factores mostraron coeficientes de consistencia interna adecuados.

\section{PALABRAS CLAVE}

Roles de género, adaptación cultural, propiedades psicométricas, medición, docentes universitarios

\section{ABSTRACT}

This study aimed to analyze the psychometric properties of cultural adaptation of the gender role scale in university teachers. Method: It's descriptive and instrumental quantitative study, in which 232 teachers from a university in Colombia participated. Internal consistency and validity analyzes of the scale factors were performed. Results: Throughout the adaptation procedure, the adjustments made were considered pertinent both in the analysis of judges and in advanced driving. The factorial structure of three factors explained $64.677 \%$ of the variance, although changes in the location of three items were evident, which leads to a theoretical discussion of such factors. The scale and its factors showed adequate internal consistency coefficients.

\section{KEY WORDS}

Gender roles, cultural adaptation, psychometric properties, measurement, university teachers.

\section{INTRODUCCIÓN}

La categoría de género es una construcción social producto de la historia y a la vez un productor de nuevas historias, que permite comprender las relaciones sociales basadas en las diferencias de poder percibidas entre los géneros, en el que se determinan jerarquías entre hombres y mujeres en una determinada sociedad (Scott, 1986; Boff y Muraro, 2004). Cada sociedad, de acuerdo a un lugar y tiempo, establecen formas particulares para expresar pensamientos, sentimientos y comportamientos que en la mayoría de casos, se condicionan a características fisiológicas y sexuales que precisan y limitan el modo de ser hombre y ser mujer a través de roles de género, que implican que los seres humanos asuman actitudes productos del patriarcado y la masculinidad hegemónica, de esta forma, se establecen las interacciones entre hombres y mujeres, sus conflictos, representaciones de la realidad social y de intervenir en ella (Boff y Muraro, 2004).

A su vez, los roles de género son construcciones sociales, que permiten determinar las representaciones masculinas y femeninas, se ajustan a las convenciones de una determinada cultura, y pueden transformarse a través del tiempo teniendo en cuenta los diferentes contextos en los que se desenvuelve la persona, así, los roles de género designan funciones, expectativas y normas que se espera que las mujeres y los hombres cumplan en una sociedad, y dictan pautas sobre la forma como debe ser, sentir y actuar el individuo dependiendo en principio del sexo al que pertenecen (Saldívar, et. al, 2015). Dichos roles, marcan una posición específica en una estructura social y las actividades que estas lo conforman, como los comportamientos enmarcados dentro de una religión (Samani, 2016) o inclusive en situaciones asociadas a salud, como repertorios comportamentales patológicos que se gestan a partir de una creencia tradicional sobre un rol de género (Kulis, Marsiglia y Nagoshi, 2010). Lo anterior, en términos de desigualdad, ya que algunas actividades o características son socialmente valoradas como más importantes o superiores, mientras que otras se consideran inferiores o menos trascendentes, pese a que, en la actualidad, en algunos países se admiten que los roles de las mujeres y los hombres han cambiado respecto de los tiempos pasados, y en muchos de los casos, se asume que ese cambio ha favorecido a unos y ha restado privilegios a los otros (Saldívar, et. al, 2015), e incluso se 
ha favorecido la emisión de violencia basada en las tradiciones relacionadas a dichos roles (Maldonado, 2015).

Estudios a nivel internacional como los de Gilbert (1998), Spence y Buckner, (2000), Anaya y Suarez (2006), Eagly (2009), González et. al (2015), Becerra, (2015), Redlick (2018) y Fernández et.al. (2018), muestran el trabajo que se profundiza respecto a roles de género, estereotipos hacia hombres y mujeres y su relación con la participación social y política, el comportamiento y características psicológicas de la persona, la construcción de la identidad, el nivel de actividad en la vejez, el impacto en el bienestar, salud y trabajo, entre otros; todo ello, en el marco de desigualdades de género presentes en términos de condiciones de estudio, trabajo, asignación de roles, tareas y salarios en los diferentes contextos en los que está inmerso el ser humano, materializándose a modo de creencias compartidas que guían el quehacer de los individuos. Concretamente, en el contexto educativo, las instituciones de educación superior son espacios en que se relacionan factores culturales, sociales, psicológicos, filosóficos, ideológicos, históricos, familiares, económicos y políticos, que influyen en la interacción de las dinámicas individuales y grupales entre los miembros de la comunidad universitaria (docentes, estudiantes y administrativos), y las cuales pueden aportar positiva o negativamente a la convivencia en su interior (González y Mora, 2014; Zambrano, Perugache y Figueroa, 2017). Al respecto, Díaz y Rocha (2005) y López-Molina y VázquezGuerrero (2018), señalan que las universidades transfieren aspectos patriarcales de control que fomentan la superioridad del hombre sobre la mujer, por lo tanto, los estudios de género en relación con la educación constituyen un campo de investigación muy importante, debido a que da lugar a la interpretación del contexto en el que se transmiten y difunden los códigos de género que cada sociedad o grupo cultural impone y que influyen de manera determinante en la adquisición de las identidades femeninas y masculinas de los sujetos.

Por tal motivo, las instituciones de educación superior se convierten en principales agentes de socialización entre hombres y mujeres basados en la desigualdad e imponiendo unos roles de género establecidos históricamente, que siguen reproduciendo patrones comportamentales dentro y fuera del contexto universitario, como en la familia, el trabajo y las diversas esferas de la vida. Sin embargo, es necesario mencionar como algunas instituciones de educación superior debido al contexto mencionado anteriormente, han trazado la equidad de género como principio central en los diferentes estatutos y reglamentos, al igual, que la formalización de órganos de control y diversas dependencias que atiendan las denuncias de la comunidad universitaria y formulen políticas que propendan por campañas, programas y procesos en contra de la violencia, desigualdad de género y la no perpetuación de roles de género tradicionales (Ordorika, 2015; Sales-Oliveira, Villas-Boas y Las-Heras, 2016).

Específicamente, en los docentes, se reconoce la importancia que tiene el género, ya que las relaciones educativas propias en las que se ven inmersos son relaciones de comunicación que pueden contribuir a la construcción de roles de género, que no hacen sino reforzar prejuicios y estereotipos que históricamente se han asociado a cada uno de los géneros, a través de las diferentes prácticas cotidianas basadas en la violencia como formas legitimadas de reafirmación de la masculinidad, tan naturalizadas que muchas de ellas pasan desapercibidas. Estas prácticas van desde el acoso callejero, la agresión a poblaciones diversas, la segregación en las áreas de estudio, las dificultades que enfrentan las mujeres en la docencia e investigación, las brechas salariales, violencia laboral, entre 
otras (Connell, 2001; Zapata y Ayala, 2014), las cuales son necesarias abordar, considerando la importancia del bienestar docente para el fomento de su desarrollo personal y profesional como catalizador de un modelo social más benéfico (Caballero y Nieto, 2015)

De esta manera, Acuña, (2014) afirma la importancia de trabajar en estudios de género e incorporar la perspectiva de género en las universidades, a través de espacios públicos donde se construyen conocimientos, se genera reflexión, se investiga en torno a problemáticas de la realidad y se desarrollan estrategias de acción social que inciden en el contexto social. Reconociendo que un primer paso, es contar con técnicas e instrumentos contextualizados, que permitan medir un fenómeno social de manera válida y confiable, para que, a partir de la información recolectada, se puedan establecer estrategias de prevención, promoción e intervención con respaldo científico y técnico. Así, los instrumentos estandarizados, son unas de las herramientas necesarias para desarrollar procesos rigurosos de medición y evaluación, que, apoyadas de un adecuado referente teórico, permiten la toma de decisiones que se derivan en intervenciones en los diversos campos de acción en los que se encuentra inmerso el ser humano (Fernández-Ballesteros, 1996).

Propendiendo a adoptar una postura respecto a la definición teórica de las variables de estudio, se procede a una revisión de estudios internacionales, nacionales y regionales, relacionados a la construcción y adaptación de instrumentos, se encuentra que la variable de género ha sido estudiada desde diversas categorías (Castillo y Montes, 2008; Tura, Martínez, Pons y Simo, 2010; Román, Domínguez y Saucedo, 2015; Chahín y Briñez, 2015; Andrade, 2016; Valadez y Luna, 2017), pero específicamente instrumentos relacionados a roles de género en la búsqueda realizada son muy escasos. El estudio de Castillo y Montes
(2008), propone una caracterización a través de la escala del concepto de estereotipos de género, comprendidos teóricamente por los autores como aquellas creencias sobre las diferencias existentes entre las mujeres y los hombres, entendiendo que tradicionalmente, se supone, se han destinado características a las personas en función del grupo de género al que pertenecen, produciendo un cambio en las creencias o estereotipos de género a partir de un fenómeno social. Por su parte, Chahin y Briñez (2015), validan la escala de Ideología de Género en adolescentes colombianos, definido el atributo como aquellas creencias que tanto hombres como mujeres desarrollan sobre los roles y conductas en las relaciones que los sexos deben mantener entre sí. En esta misma búsqueda, se encuentran estudios relacionados a contextos educativos como el de Tura et.al. (2010), quienes en docentes y administrativos se propusieron instrumentalizar la percepción de igualdad de oportunidades entre mujeres y hombres en una institución de educación superior de España. En este mismo orden, aparece el estudio sobre cultura de género entendida como una serie de procesos administrativos, equidad laboral, prestaciones, clima laboral y autoritarismo, realizado por Román et.al. (2016) en el sector salud y educativo. Otro estudio es el de Valadez et. al. (2017) al abordar mandatos de género asociados a la normatividad comportamental del patriarcado en una población de estudiantes Universitarios de México. Asimismo, se reporta la investigación de Saldivar et.al. (2015), que enfatiza en los roles de género, una variable referida hacia aquellas verbalizaciones que normalmente el colectivo social asocia hacia las características comportamentales propias de un individuo por el hecho que su género sea masculino o femenino. Ante ello, se propuso una escala de medición para el atributo en población mexicana.

Por lo anterior, al realizar la exploración de los antecedentes que se han gestado respecto a la 
construcción y adaptación de instrumentos que midan los roles de género o constructos similares, se evidencian diferentes publicaciones (Castillo y Montes, 2008); Tura, Martínez, Pons y Simo, 2010; Román, Domínguez y Saucedo, 2016; Chahín y Briñez, 2015; Andrade, 2016; Valadez y Luna, 2017), las cuales presentan como objetivo la realización o la adaptación transcultural de instrumentos de medición psicológica con la pretensión de caracterizar constructos tales como: mandatos de género, cultura de género, igualdad de oportunidades de género, ideología de género, estereotipos de género y entre otros conceptos. Sin embargo, ningún estudio consultado se relaciona directamente con roles de género en contexto universitario, puesto que las pruebas mencionadas, si bien se dirigen a poblaciones estudiantiles, docentes y personas en general, principalmente abordan a habitantes de países tales como España, México y Brasil.

Así pues, se propuso realizar una investigación similar en aras de la adaptación transcultural del instrumento de Saldivar et.al. (2015) para el contexto de docentes universitarios, a diferencia del estudio original. Es de mencionar que Saldivar et.al. (2015) tiene como pretensión principal, caracterizar los roles de género en población mexicana en general. Más aun, el instrumento original no pretende describir la variable en función de un contexto de educación superior; de ahí la necesidad de adaptación de un test que responda a medir roles de género en docentes universitarios, debido a la transmisión cultural de dichos roles de género junto con sus conductas esperadas ocurren en los diferentes escenarios de interacción de las personas, y así, el contexto educativo, como uno de los ámbitos más importantes para reproducción de creencias y características culturales, sociales, económicas y políticas (Acker, 2003; Páramo, 2010).

Finalmente, este artículo presenta los resultados del proceso de adaptación transcultural del cuestionario de roles de género, haciendo énfasis en el proceso desarrollado y las propiedades psicométricas obtenidas, esto demostrado en el proceso de teorización a la luz de los antecedentes encontrados, el vacío de conocimiento y la necesidad de investigación al respecto, la metodología empleada para la adaptación del instrumento, los resultados obtenidos y la discusión construida a partir de las evidencias de validez y confiabilidad, análisis factorial y construcción de baremos.

\section{MÉTODO}

Se realizó un estudio cuantitativo de carácter descriptivo e instrumental (Montero \& León, 2007), en tanto el propósito principal fue adaptar la escala de roles de género de Saldivar et.al (2015) en docentes universitarios, entendiendo la adaptación como el proceso de traducción, ajuste cultural e idiomático y la comprobación de las características psicométricas de fiabilidad y validez (Escobar, 2004).

\section{PARTICIPANTES}

Participaron 232 docentes de una universidad pública del sur occidente colombiano. De los cuales $151(65 \%)$ fueron hombres y $81(35 \%)$ mujeres. Respecto a los rangos de edad, el $8,6 \%$ se encuentra entre los 18 y 29 años, el $22,8 \%$ entre los 30 y 39 años, el $24,1 \%$ entre los 40 a 49 años, el $24,1 \%$ entre los 50 y 59 años y el $12,1 \%$ de los participantes cuenta con más de 60 años. Participaron docentes de las 11 facultades con las cuales cuenta la Universidad.

\section{INSTRUMENTO Y PROCEDIMIENTO}

La escala a validar se denomina Escala de Roles de Género (Saldívar, et al., 2015). Esta consta de 18 reactivos tipo Likert, con 5 opciones de respuesta (1=Totalmente en desacuerdo a $5=$ Totalmente de acuerdo), agrupados en tres factores, a saber: a) roles masculinos estereotipados, b) roles femeninos 
estereotipados y c) roles tradicionales para mujeres y varones $(6,7$ y 5 reactivos respectivamente), con los que se busca recoger la percepción de las y los participantes sobre afirmaciones que hacen referencia a roles, características, habilidades y tareas particulares, consideradas como propias de varones y mujeres. Dicho instrumento fue desarrollado con personas provenientes de diversos estados de México, mostrando ser alta consistencia interna (alfa de Cronbach de .89 para los 18 reactivos), y una varianza total explicada del $50.8 \%$.

El proceso de adaptación inició con la solicitud formal a los autores de instrumento para la realización del proceso. Con el aval de los autores, se procedió a la revisión y ajuste de algunos ítems por parte de los investigadores teniendo en cuenta el contexto universitario (en el rol de la docencia) y colombiano al cual se adaptaría. Posteriormente, la escala, fue valorada por 3 jueces expertos en psicometría, en el atributo (roles de género) y en el contexto universitario. Después, se sometió la escala a prueba piloto con 30 particionaste, y finalmente se aplicó a la población objeto de estudio a través de un aplicativo virtual, realizando un muestreo intencional.

\section{ASPECTOS ÉTICOS Y ANÁLISIS DE RESULTADOS}

La investigación se realizó bajo la Resolución 8430 de 1993 y la Ley 1090 de 2006 de Colombia, donde se garantizó que el manejo de información fue estrictamente confidencial, además de que el estudio fue catalogado de riesgo mínimo, al no propender cambios del comportamiento humano y afectar la integridad física de los participantes. Asimismo, la investigación contó con el aval del Comité de Ética en Investigaciones de la Universidad, con la previa autorización de la población a través de la firma de un consentimiento informado y el aval y apoyo de la Vicerrectoría de Investigación e Interacción Social, la Vicerrectoría Académica y la División de Acreditación Institucional de la Universidad.

Una vez recolectada la información, se realizaron los análisis psicométricos en el paquete estadístico SPSS, versión 23. Se retoman las evaluaciones por parte de los jueces, así como los datos recabados en el proceso de pilotaje. Se realizó un análisis factorial exploratorio AFE de los ítems, tanto para el total de la muestra como por subgrupos (estrategia de validación del AFE). Se calcularon índices de consistencia interna tanto para la escala total como para subescalas, así como la correlación ítem test (índice de discriminación).

\section{RESULTADOS}

En la fase inicial de la investigación se procedió a realizar ajustes de forma a algunos ítems de la escala, manteniendo su contenido y correspondencia con el atributo. En la tabla 1 se muestran los ítems en su versión original y la modificación realizada.

Tabla 1. Ítems modificados

\begin{tabular}{ll}
\hline Ítem original & Ítem ajustado \\
\hline $\begin{array}{l}\text { Ítem 14. Un hombre verdadero no muestra sus } \\
\text { debilidades }\end{array}$ & $\begin{array}{l}\text { 14. Un hombre de verdad no muestra sus } \\
\text { debilidades }\end{array}$ \\
\hline $\begin{array}{l}\text { Ítem 15. Un hombre verdadero no muestra sus } \\
\text { sentimientos. }\end{array}$ & $\begin{array}{l}\text { I5. Un hombre de verdad no muestra sus } \\
\text { sentimientos. }\end{array}$ \\
\hline $\begin{array}{l}\text { Ítem 3. Una mujer se realiza hasta que se } \\
\text { convierte en madre } 3 . \text { Una mujer se realiza cuando se convierte } \\
\text { en madre }\end{array}$ \\
\hline
\end{tabular}


Para la validación de contenido y de los ajustes realizados a la escala, se contó con la evaluación realizada por 3 jueces de las áreas de psicometría, género y docencia universitaria. Tales jueces cumplían con las características de experticia, disponibilidad e imparcialidad mencionados por Escobar y Cuervo (2008). Los criterios evaluados fueron claridad, pertinencia y equivalencia semántica, los cuales fueron calificados de 1 a 3 ( 1 si el ítem no cumplía, 2 si cumplía, pero se debían hacer ajustes y 3 si el ítem cumplía totalmente). Así, todos los ítems obtuvieron promedios de 3 en las calificaciones de los criterios, por lo cual se asumió tanto su idoneidad para medir el atributo como la pertinencia de los ajustes realizados. Posteriormente, se realizó un pilotaje para evaluar la claridad de los ítems. Se contó con la participación de 30 docentes universitarios, quienes calificaron al ítem como claro/no claro. En el pilotaje, todos los ítems tuvieron un porcentaje de claridad superior al $87 \%$ (el criterio mínimo establecido fue del $85 \%$, porcentajes menores ameritaban ajustes al ítem).

Una vez recogidos los datos de la aplicación general, se procedió a realizar los análisis psicométricos. En la prueba de KMO se obtuvo una medida de 0,913 y la esfericidad de Bartlett fue de 2739,246 (Sig. 0,000), lo cual permitió la realización del análisis factorial exploratorio de la escala (AFE). El AFE se hizo mediante el método de extracción de componentes principales, con una rotación oblicua de tipo oblimin que se utiliza cuando se supone que los ítems no serán totalmente independientes y los factores resultantes podrían tener alta covarianza (Soret, 2007). La matriz rotada convergió en 5 iteraciones, obteniéndose 3 factores que explican el $64,677 \%$ de la varianza total explicada. Las comunalidades (h2) de los datos oscilaron entre .472 a .823 , valores considerados óptimos. En la tabla 2 se muestran los factores identificados, las cargas factoriales de los ítems y la comunalidad (h2) de cada ítem.

\begin{tabular}{|c|c|c|c|c|}
\hline \multicolumn{4}{|c|}{$\begin{array}{c}\text { Tabla } 2 . \\
\text { Análisis factorial exploratorio }\end{array}$} & \multirow{3}{*}{ h2 } \\
\hline \multirow[b]{2}{*}{ Ítem } & \multicolumn{3}{|c|}{ Componente } & \\
\hline & SPRM & SPRF & SPRMF & \\
\hline 15. Un hombre necesita de varias parejas sexuales. & ,624 & & & ,761 \\
\hline $\begin{array}{l}\text { 16. Una familia funciona mejor si el hombre establece las } \\
\text { reglas. }\end{array}$ & ,457 & & & ,775 \\
\hline 17. Un hombre es más racional que una mujer. & ,742 & & & ,472 \\
\hline 113. Un hombre es infiel por naturaleza. & 667 & & & ,665 \\
\hline 114. Un hombre de verdad NO muestra sus debilidades. & ,835 & & &, 574 \\
\hline I15.Un hombre de verdad NO muestra sus sentimientos. & ,741 & & & ,634 \\
\hline 116. Un hombre es más agresivo que una mujer. & ,479 & & & ,642 \\
\hline I17. La infidelidad es imperdonable en una mujer. & ,673 & & & ,639 \\
\hline $\begin{array}{l}\text { 11. La mujer tiene mayor capacidad de cuidar a los } \\
\text { enfermos. }\end{array}$ & & ,833 & & ,627 \\
\hline 12. Una madre es más cariñosa que un padre. & & ,835 & & ,739 \\
\hline $\begin{array}{l}\text { 14. Una mujer posee mayor fortaleza emocional que un } \\
\text { hombre. }\end{array}$ & & ,779 & & ,706 \\
\hline 18. Un hombre es menos sensible que una mujer. & & ,482 & & ,663 \\
\hline $\begin{array}{l}\text { 112. Los hijos y las hijas son mejor educados por una madre } \\
\text { que por un padre. }\end{array}$ & & ,469 & & ,628 \\
\hline I3. Un hombre es infiel por naturaleza. & & & ,498 & ,823 \\
\hline
\end{tabular}




\begin{tabular}{|c|c|c|}
\hline $\begin{array}{l}\text { 19. La mujer tiene habilidades innatas para el quehacer } \\
\text { doméstico. }\end{array}$ & ,493 & ,676 \\
\hline 110. Una buena mujer debe atender a su pareja. & ,784 & ,504 \\
\hline I11. El hombre es más hábil que la mujer para cortejar. & 721 &, 578 \\
\hline I18. El hombre debe proteger a la familia. & 640 &, 538 \\
\hline \multicolumn{3}{|c|}{$\begin{array}{l}\text { *SPRM hace referencia a la dimensión identificada como Sistema patriarcal y roles de género } \\
\text { masculinos, SPRF hace referencia a la dimensión identificada como Sistema patriarcal y roles de } \\
\text { género femeninos, y SPRMF indica la dimensión sistema patriarcal y roles de género masculinos y } \\
\text { femeninos. }\end{array}$} \\
\hline
\end{tabular}

Respecto a la correlación ítem - test, se encontró que todos los ítems poseen una correlación positiva significativa con la puntuación total de la escala, como se puede ver en la tabla 3. Los resultados identificados parecen indicar que los puntajes obtenidos en cada ítem implican desempeños diferenciales en la puntuación global, en tanto poseen alta relación con el desempeño general en la prueba.

Tabla 3. Correlación ítem-test

\begin{tabular}{ll}
\hline Ítem & Índice Correlación \\
\hline Item1 &, $685^{* *}$ \\
\hline Item2 &, $701^{* *}$ \\
\hline Item3 &, $656^{* *}$ \\
\hline Item4 &, $635^{* *}$ \\
\hline Item5 &, $526^{* *}$ \\
\hline Item6 &, $681^{* *}$ \\
\hline Item7 &, $691^{* *}$ \\
\hline Item8 &, $750^{* *}$ \\
\hline Item9 &, $788^{* *}$ \\
\hline Item10 &, $724^{* *}$ \\
\hline Item11 &, $725^{* *}$ \\
\hline Item12 &, $814^{* *}$ \\
\hline Item13 &, $750^{* *}$ \\
\hline Item14 &, $781^{* *}$ \\
\hline Item15 &, $734^{* *}$ \\
\hline Item16 &, $727^{* *}$ \\
\hline Item17 &, $681^{* *}$ \\
\hline Item18 &, $601^{* *}$ \\
\hline
\end{tabular}


En cuanto a la relación entre dimensiones, se encontraron correlaciones positivas moderadas entre los factores, como se muestra en la tabla 4.

Tabla 4. Correlación de Spearman entre factores

\begin{tabular}{llll} 
& SPRF & SPRM & SPRMF \\
\hline SPRF & ----- & & \\
\hline SPRM &, 667 & ----- & \\
\hline SPRMF &, 663 &, 663 & ---- \\
\hline
\end{tabular}

*SPRM hace referencia a la dimensión identificada como Sistema patriarcal y roles de género masculinos, SPRF hace referencia a la dimensión identificada como Sistema patriarcal $y$ roles de género femeninos, y SPRMF indica la dimensión sistema patriarcal y roles de género masculinos y femeninos.

Como plantea Bisquerra (1989), una forma de validar los hallazgos identificados a través del AFE es comparar las soluciones factoriales entre subgrupos de la población. Con tal consideración, se realizaron dos AFE, bajo los mismos parámetros, en los subgrupos de mujeres y hombres participantes. En ambas soluciones, se encontraron tres factores con valores propios mayores a 1 , a la vez que la varianza explicada varió entre el $65 \%$ al $66 \%$, con lo que cual existen evidencias para presumir la importancia explicativa de los tres factores hallados (ver tabla 5).
Tabla 5. Estadísticos del AFE entre hombres y mujeres

\begin{tabular}{lll}
\hline Estadístico & Mujeres & Hombres \\
\hline Comunalidad $\min$ & .550 & .486 \\
\hline Comunalidad max & .889 & .790 \\
\hline $\begin{array}{l}\text { Componentes con } \\
\text { valores propios }\end{array}$ & 3 & 3 \\
\hline $\begin{array}{l}\text { Varianza total } \\
\text { explicada }\end{array}$ & $65,735 \%$ & $66,821 \%$ \\
\hline
\end{tabular}

Referido a la confiabilidad de la escala, esta se estimó mediante el coeficiente de consistencia interna Alpha de Cronbach. Se encontraron coeficientes óptimos tanto en la totalidad del instrumento como en sus factores: el valor del Alpha de Cronbach para la escala total fue de .935; para el factor Roles Masculinos Estereotipados fue de 8,96; para el factor Roles Femeninos Estereotipados fue de 8,66 y para el factor Roles Tradicionales para Mujeres y Varones fue de 8,24.

En la tabla 6, se resumen los datos relacionados con la tendencia de las respuestas, y los valores de asimetría y curtosis de la distribución por cada ítem. 
Tabla 6. Asimetría, curtosis y frecuencia de respuestas

\begin{tabular}{|c|c|c|c|c|c|c|c|c|}
\hline \multicolumn{2}{|c|}{ Ítems } & As & $K$ & $\begin{array}{l}1 \\
\mathrm{Fr} / \%\end{array}$ & $\begin{array}{l}2 \\
\mathrm{Fr} / \%\end{array}$ & $\begin{array}{l}3 \\
\mathrm{Fr} / \%\end{array}$ & $\begin{array}{l}4 \\
\mathrm{Fr} / \%\end{array}$ & \begin{tabular}{|l|}
5 \\
$\mathrm{Fr} / \%$
\end{tabular} \\
\hline I-1 & $\begin{array}{l}\text { Un hombre } \\
\text { necesita de varias } \\
\text { parejas sexuales. }\end{array}$ & -.036 & -1.116 & $\begin{array}{c}53 \\
(22,8 \%)\end{array}$ & $\begin{array}{c}29 \\
(12,5 \%)\end{array}$ & $\begin{array}{c}70 \\
(30,2 \%)\end{array}$ & $\begin{array}{c}46 \\
(19,8 \%)\end{array}$ & $\begin{array}{c}34 \\
(14,7 \%)\end{array}$ \\
\hline $1-2$ & $\begin{array}{l}\text { Una familia } \\
\text { funciona mejor si el } \\
\text { hombre establece } \\
\text { las reglas. }\end{array}$ & .093 & -1.139 & $51(22 \%)$ & $44(19 \%)$ & $\begin{array}{c}(60 \\
(25,9 \%)\end{array}$ & $44(19 \%)$ & $\begin{array}{c}33 \\
(14,2 \%)\end{array}$ \\
\hline $1-3$ & $\begin{array}{l}\text { Un hombre es más } \\
\text { racional que una } \\
\text { mujer. }\end{array}$ & .973 & -.058 & $\begin{array}{c}110 \\
(47,4 \%)\end{array}$ & $\begin{array}{c}48 \\
(20,7 \%)\end{array}$ & $44(19 \%)$ & $\begin{array}{c}16 \\
(6,9 \%)\end{array}$ & $14(6 \%)$ \\
\hline $1-4$ & $\begin{array}{l}\text { Un hombre es infiel } \\
\text { por naturaleza. }\end{array}$ & .134 & -.945 & $\begin{array}{c}53 \\
(22,8 \%)\end{array}$ & $44(19 \%)$ & $\begin{array}{c}73 \\
(31,5 \%)\end{array}$ & $\begin{array}{c}41 \\
(17,7 \%)\end{array}$ & $\begin{array}{c}21 \\
(9,1 \%)\end{array}$ \\
\hline $1-5$ & $\begin{array}{l}\text { Un hombre de } \\
\text { verdad NO muestra } \\
\text { sus debilidades. }\end{array}$ & 1.506 & 1.719 & $\begin{array}{c}145 \\
(62,5 \%)\end{array}$ & $\begin{array}{c}41 \\
(17,7 \%)\end{array}$ & $\begin{array}{c}35 \\
(15,1 \%)\end{array}$ & $6(2,6 \%)$ & $5(2,2 \%)$ \\
\hline I-6 & $\begin{array}{l}\text { Un hombre de } \\
\text { verdad NO muestra } \\
\text { sus sentimientos. }\end{array}$ & 1.234 & .796 & $\begin{array}{c}129 \\
(55,6 \%)\end{array}$ & $\begin{array}{c}45 \\
(19,4 \%)\end{array}$ & $\begin{array}{c}42 \\
(18,1 \%)\end{array}$ & $9(3,9 \%)$ & $7(3 \%)$ \\
\hline $1-7$ & $\begin{array}{l}\text { Un hombre es más } \\
\text { agresivo que una } \\
\text { mujer. }\end{array}$ & 1.301 & .691 & $\begin{array}{c}139 \\
(59,9 \%)\end{array}$ & $\begin{array}{c}37 \\
(15,9 \%)\end{array}$ & $\begin{array}{c}34 \\
(14,7 \%)\end{array}$ & $\begin{array}{c}15 \\
(6,5 \%)\end{array}$ & $7(3 \%)$ \\
\hline I-8 & $\begin{array}{l}\text { La infidelidad es } \\
\text { imperdonable en } \\
\text { una mujer. }\end{array}$ & .835 & -.207 & $\begin{array}{c}105 \\
(45,3 \%)\end{array}$ & $\begin{array}{c}61 \\
(26,3 \%)\end{array}$ & $\begin{array}{c}46 \\
(19,8 \%)\end{array}$ & $\begin{array}{c}17 \\
(7,3 \%)\end{array}$ & $3(1,3 \%)$ \\
\hline $1-9$ & $\begin{array}{l}\text { La mujer tiene } \\
\text { mayor capacidad } \\
\text { de cuidar a los } \\
\text { enfermos. }\end{array}$ & 1.127 & .411 & $\begin{array}{c}124 \\
(53,3 \%)\end{array}$ & $\begin{array}{c}46 \\
(19,8 \%)\end{array}$ & $\begin{array}{c}42 \\
(18,1 \%)\end{array}$ & $\begin{array}{c}13 \\
(5,6 \%)\end{array}$ & $7(3 \%)$ \\
\hline $\mathrm{I}-10$ & $\begin{array}{l}\text { Una madre es más } \\
\text { cariñosa que un } \\
\text { padre. }\end{array}$ & .617 & -.681 & $\begin{array}{c}105 \\
(45,3 \%)\end{array}$ & $\begin{array}{c}37 \\
(15,9 \%)\end{array}$ & $\begin{array}{c}57 \\
(24,6 \%)\end{array}$ & $\begin{array}{c}24 \\
(10,3 \%)\end{array}$ & $9(3,9 \%)$ \\
\hline $\mathrm{I}-11$ & \begin{tabular}{|l|} 
Una mujer posee \\
mayor fortaleza \\
emocional que un \\
hombre.
\end{tabular} & .580 & -.926 & $\begin{array}{c}105 \\
(45,3 \%)\end{array}$ & $\begin{array}{c}43 \\
(18,5 \%)\end{array}$ & $\begin{array}{c}57 \\
(24,6 \%)\end{array}$ & $\begin{array}{c}25 \\
(10,8 \%)\end{array}$ & $2(.9 \%)$ \\
\hline $\mid-12$ & $\begin{array}{l}\text { Un hombre es } \\
\text { menos sensible } \\
\text { que una mujer. }\end{array}$ & .786 & -.296 & $\begin{array}{c}107 \\
(46,1 \%)\end{array}$ & $\begin{array}{c}49 \\
(21,1 \%)\end{array}$ & $\begin{array}{c}56 \\
(24,1 \%)\end{array}$ & $\begin{array}{c}15 \\
(24,1 \%)\end{array}$ & $5(2,2 \%)$ \\
\hline
\end{tabular}




\begin{tabular}{|l|l|c|c|c|c|c|c|c|}
\hline I-13 & $\begin{array}{l}\text { Los hijos y las } \\
\text { hijas son mejor } \\
\text { educados por una } \\
\text { madre que por un } \\
\text { padre. }\end{array}$ & 1.045 & .116 & $\begin{array}{c}127 \\
(54,7 \%)\end{array}$ & $\begin{array}{c}40 \\
(17,2 \%)\end{array}$ & $\begin{array}{c}46 \\
(19,8 \%)\end{array}$ & $14(6 \%)$ & $5(2,2 \%)$ \\
\hline I-14 & $\begin{array}{l}\text { Un hombre es infiel } \\
\text { por naturaleza. }\end{array}$ & 1.471 & 1.373 & $\begin{array}{c}143 \\
(61,6 \%)\end{array}$ & $43(18,5)$ & $\begin{array}{c}29 \\
(12,5 \%)\end{array}$ & $\begin{array}{c}12 \\
(5,2 \%)\end{array}$ & $5(2,2 \%)$ \\
\hline I-15 & $\begin{array}{l}\text { La mujer tiene } \\
\text { habilidades innatas } \\
\text { para el quehacer } \\
\text { doméstico. }\end{array}$ & 1.384 & 1.074 & $\begin{array}{c}143 \\
(61,6 \%)\end{array}$ & $\begin{array}{c}38 \\
(16,4 \%)\end{array}$ & $\begin{array}{c}35 \\
(15,1 \%)\end{array}$ & $11(4,7 \%)$ & $5(2,2 \%)$ \\
\hline I-16 & $\begin{array}{l}\text { Una buena mujer } \\
\text { debe atender a su } \\
\text { pareja. }\end{array}$ & .486 & -.932 & $\begin{array}{c}92 \\
(39,7 \%)\end{array}$ & $44(19 \%)$ & $\begin{array}{c}59 \\
(25,4 \%)\end{array}$ & $\begin{array}{c}31 \\
(13,4 \%)\end{array}$ & $6(2,6 \%)$ \\
\hline I-17 & $\begin{array}{l}\text { El hombre es más } \\
\text { hábil que la mujer } \\
\text { para cortejar. }\end{array}$ & 1.285 & .985 & $\begin{array}{c}130 \\
(56 \%)\end{array}$ & $\begin{array}{c}43 \\
(18,5 \%)\end{array}$ & $44(19 \%)$ & $6(2,6 \%)$ & $9(3,9 \%)$ \\
\hline I-18 & $\begin{array}{l}\text { El hombre debe } \\
\text { proteger a la } \\
\text { familia. }\end{array}$ & -.182 & -1.353 & $\begin{array}{c}54 \\
(23,3 \%)\end{array}$ & $\begin{array}{c}(12,1 \%) \\
28\end{array}$ & $44(19 \%)$ & 55 & $51(22 \%)$ \\
\hline
\end{tabular}

Finalmente, respecto a la baremación del instrumento para la población docente en la región, se retomaron los puntos de corte establecidos por los cuartiles de la distribución de respuestas, como se presenta en la tabla 7.

Tabla 7. Baremos para la población

\begin{tabular}{ll}
\hline \multicolumn{1}{c}{ Puntuación } & \multicolumn{1}{c}{ Interpretación } \\
\hline Menor a 26 & Percepción estereotipada baja de roles de género \\
\hline Entre 27 y 37 & Percepción moderadamente estereotipada de roles de género \\
\hline Entre 38 y 49 & Percepción considerablemente estereotipada de roles de género \\
\hline Superior a 49 & Percepción altamente estereotipada de roles de género \\
\hline
\end{tabular}

\section{DISCUSIÓN}

El objetivo de la presente investigación fue realizar la adaptación de la escala de roles de género en docentes universitarios, $y$ analizar sus propiedades psicométricas. Al momento de investigar en psicología, una de las situaciones metodológicas que todo investigador se enfrenta es seleccionar un adecuado instrumento de medida que no sólo responda de manera correcta a la variable de estudio trabajada, sino que además dicha herramienta disponga de las propiedades psicométricas de validez y confiabilidad que garanticen al estudio, la obtención de una medición exacta, que corresponda a las realidades de los individuos y grupos, de acuerdo a sus características 
culturales y contextuales (Escobar, 2004). Así, a la hora de identificar un instrumento, se cuenta con dos opciones: su construcción o su adaptación. La primera comprende desarrollar un instrumento nuevo, teniendo en cuenta los recursos económicos, logísticos y de tiempo que dicha tarea implica. La segunda alternativa, es la adaptación de instrumentos construidos en otros contextos, a partir de un proceso de traducción lingüística que economiza trabajo y tiempo (Escobar, 2004; Lira y Caballero, 2020).

Lira y Caballero (2020) sostienen que, a pesar de que el español es el segundo idioma más hablado en el mundo, se presentan ciertas particularidades lingüísticas, como dialectos o el uso de léxico diferente, según las regiones donde se encuentren los hispano-hablantes. Por lo tanto, esta situación justifica la importancia de generar procesos de adaptaciones transculturales en un mismo idioma, y más sobre temas relacionados al género como concepto de compleja medición que depende del sistema de prácticas, representaciones, normas y valores propios de una sociedad que se elaboran desde las particularidades biológicas y culturales que presentan los seres humanos (Arcos, et.al, 2006). Acotando a lo anteriormente expuesto, contar con instrumentos específicos de roles de género responde a la necesidad y a los cambios del contexto, donde se encuentran realidades como el aumento de la participación de las mujeres en variados escenarios como el laboral y educativo, y la necesidad de identificar y medir dichas transformaciones en relación a las posibles consecuencias físicas y psicológicas de los individuos inmersos en un sistema predominantemente patriarcal (Román, Domínguez, Saucedo y Tánori, 2016).

Por lo anterior, aportar con instrumentos estandarizados y actualizados para medir atributos como los roles de género, se convierten en una herramienta útil para que interesados en el tema puedan medir dicho atributo, y de esta manera, desde una lectura crítica y articulada a los procesos de evaluación, se puedan tomar decisiones de prevención e intervención ante la violencia, desigualdad de género y la no perpetuación de roles de género tradicionales. Este instrumento aporta a una mirada cuantitativa, que permita que los diferentes órganos de control y diversas dependencias universitarias diagnostiquen $\mathrm{y}$ atiendan las denuncias de los actores que hacen parte de las instituciones de educación superior, en sintonía con la formulación de políticas, programas y campañas contextualizadas entre docentes, estudiantes, administrativos y directivos (Ordorika, 2015; Sales-Oliveira, Villas-Boas y Las-Heras, 2016).

Respecto a las propiedades psicométricas del instrumento y respondiendo a la afirmación con la que se inicia este apartado, y en relación a la validez de contenido, las calificaciones realizadas por los jueces a los ítems modificados mostraron la pertinencia de los ajustes para medir el atributo en la población docente del contexto, cumpliéndose con el supuesto de que los ítems del test se consideran como una muestra representativa y coherente de los roles de género, y que continúan midiendo el mismo atributo incluso con los cambios realizados (Magnusson, 1975: Kerlinger y Lee, 2002; Escobar y Cuervo, 2008). Por otro lado, el pilotaje adelantado mostró que existía claridad en los ítems de la versión ajustada del instrumento, y que la comprensión de los mismos por parte de una muestra similar al grupo de estudio posterior era adecuada: esto se corresponde con el criterio mencionado por Muñiz y Fonseca (2019), relacionado con la importancia de adelantar estudios piloto para detectar, evitar y corregir posibles errores. Ambas evidencias aportan a la consideración de las diferencias contextuales para la adaptación de instrumentos de medición psicológica. Es así como los resultados obtenidos en dichos procedimientos permitieron proceder, con un 
apoyo basado en evidencias, a la aplicación del instrumento y los posteriores análisis de los datos obtenidos.

Por su parte, el análisis factorial es entendido como un modelo estadístico que representa las relaciones entre un conjunto de variables (Ferrando y Anguiano, 2010). De esta manera, la escala original de roles de género de Saldivar et.al. (2015) reportó un análisis factorial exploratorio de tres factores. EI primero denominando "roles masculinos estereotipados" que refieren características adjudicadas típicamente a los hombres (por ejemplo: ser fuertes, poco sensibles, racionales, quienes representan a la autoridad, infieles y mujeriegos); el segundo "roles femeninos estereotipados" definiendo las características consideradas como femeninas (por ejemplo: ser cariñosa, con fortaleza emocional, capaz de cuidar a los enfermos, buena educadora de hijas e hijos, y quien se realiza al convertirse en madre); y finalmente, el tercer factor, "roles tradicionales para mujeres y varones" se relaciona con la obligación de las mujeres de atender a sus parejas, y sus habilidades para la realización de tareas domésticas, mientras que para los hombres se destaca su rol de protector y habilidades para el cortejo amoroso.

Así, el análisis factorial del presente estudio obtuvo tres factores con una distribución similar en sus ítems como se muestra en la tabla 8.

Tabla 8. Comparación entre el Análisis factorial de la prueba original y adaptada.

\begin{tabular}{llll}
\hline Factores originales & Ítems Originales & Ítems Adaptados & Factores adaptados \\
\hline $\begin{array}{l}\text { Roles masculinos } \\
\text { estereotipados }\end{array}$ & $5,6,7,13,14,15$ & $5,6,7,13,14,15,16, \begin{array}{l}\text { Sistema patriarcal y roles } \\
\text { de género masculinos. }\end{array}$ \\
\hline $\begin{array}{l}\text { Roles femeninos } \\
\text { estereotipados }\end{array}$ & $1,2,3,4,8,12,16$ & $1,2,4,8,12$ & $\begin{array}{l}\text { Sistema patriarcal y roles } \\
\text { de género femeninos. }\end{array}$ \\
\hline $\begin{array}{l}\text { Roles tradicionales para } \\
\text { mujeres y varones }\end{array}$ & $9,10,11,17,18$ & $3,9,10,11,17,18$ & $\begin{array}{l}\text { Sistema patriarcal y roles } \\
\text { de género masculinos y } \\
\text { femeninos. }\end{array}$ \\
\hline
\end{tabular}

En este punto, se parte en entender que los roles de género son construcciones culturales que determinada sociedad establece en el marco del dominio del patriarcado que perpetua la diferenciación, inferioridad y subordinación entre los géneros, atribuyendo una serie de actitudes dependiendo del sexo de la persona, por lo que determinado individuo acoge una serie de pensamientos, emociones y comportamientos que lo irán formando como hombre o mujer, reflejando ante la sociedad su papel e identidad (Matos, 2014; Zambrano, Perugache y Figueroa, 2016). Por lo anterior, en el presente artículo se establecen tres definiciones de acuerdo al análisis factorial reportado, la teoría de roles de género (Butler, 2001; Boff y Muraro, 2004; Saldivar et.al., 2015) y el estudio de la escala original de roles de género de Saldivar et.al. (2015), encontrando:

Sistema patriarcal y roles de género masculinos: Según el sistema patriarcal sobresale una categoría de poder que se ejemplifica en la imposición de unas reglas que establecen entre los hombres y las mujeres, que permiten develar una serie actitudes representadas a través de roles de género frente a la sexualidad, por ejemplo, los hombres por naturaleza son promiscuos e infieles; además de la dificultad para expresar sus sentimientos, la necesidad de 
visibilizar su fuerza y racionalidad e invisibilizar sus debilidades.

Sistema patriarcal y roles de género femeninos: Según el sistema patriarcal sobresale una categoría de poder que se ejemplifica en la imposición de unas reglas que establecen entre los hombres y las mujeres, que permiten develar una serie actitudes representadas a través de roles de género que muestran a la mujer con mayores capacidades para expresar sus sentimientos, emociones y cariño, dedicándose a actividades como la educación de sus hijos, el cuidado del hogar y de los enfermos.

\section{Sistema patriarcal y roles de género masculinos y} femeninos: Según el sistema patriarcal sobresale una categoría de poder que se ejemplifica en la imposición de unas reglas que establecen entre los hombres y las mujeres, que permiten develar una serie actitudes representadas a través de roles de género relacionadas a la obligación de las mujeres de atender a sus pareja, realizar los quehaceres domésticos y sus necesidad de convertirse en madre; mientras que para los hombres se destaca su rol de protector $y$ habilidades para el cortejo amoroso.

En lo que respecta a la confiabilidad, el coeficiente Alpha de Cronbach de la escala total permite asumir que el instrumento es una herramienta que brinda la posibilidad de realizar mediciones exactas del atributo de roles de género (Quero, 2010). Por otro lado, la obtención de puntajes altos en los coeficientes de cada subescala indica que los ítems que las componen conservan homogeneidad entre sí, midiendo la misma dimensión, y por tanto aportan exactitud a las estimaciones obtenidas (Ledesma, Molina y Valero, 2002; Cervantes, 2005; Quero, 2010). Claramente, cuando se menciona que el instrumento presentado posee un nivel de confiabilidad alto, se hace referencia a la exactitud de la medida obtenida con la población objetivo: la fiabilidad hace referencia no al instrumento, sino a los puntajes que con él se obtuvo, de ahí que se recalque la importancia de la adaptación y validación de los instrumentos con las nuevas poblaciones donde quiera ser usado (Morales, 2008).

Finalmente, a partir de los datos obtenidos en el presente estudio, se propone una baremación para la interpretación de los resultados del instrumento, tomando como punto de corte los cuartiles de la distribución de respuestas, y asignando niveles de bajo, moderado, considerable y alto a tales puntos de corte. Este procedimiento obedece a la necesidad de establecer puntos de comparación de puntajes individuales con un grupo de referencia que comparta características similares, y mediante los cuales se pueda realizar análisis e interpretaciones efectivas (Magnusson, 1975; Morales, 2008).

\section{CONCLUSIONES}

Los resultados arrojados a lo largo del estudio dan cabida a afirmar que la adaptación transcultural de la Escala de Roles de Género se constituye como un instrumento eficiente en la población de docentes universitarios para la medición del atributo que se propone, comprendido en función de las percepciones que se pueda tener frente a los mismos.

Haciendo precisión en la validación del cuestionario, se concluye que es favorable para la medición de la variable en la población, dada la aprobación del proceso de validación por jueces en un primer momento, como mecanismo de verificación de la pertinencia en el contexto que llegaron a tener las modificaciones lingüísticas en los reactivos, constatando además que el atributo que se desea medir sigue siendo el mismo aun con las transformaciones en los ítems. Esto también sumado al hecho de que el pilotaje que existía la claridad y la comprensión indicada en los reactivos, articulándose un aspecto para considerar óptima la validez de la adaptación transcultural. 
En cuanto al análisis factorial, el presente estudio obtuvo tres factores con una distribución similar en sus ítems a la prueba original, más aún, se nombran y definen los nuevos factores (Sistema patriarcal y roles de género masculinos, Sistema patriarcal y roles de género femeninos, y Sistema patriarcal y roles de género masculinos y femeninos) considerando como el sistema patriarcal al que está inmerso el ser humano, impone unas reglas que se establecen entre los hombres y las mujeres, que permiten develar una serie de actitudes representadas a través de roles de género frente a la sexualidad, expresión de sentimientos, emociones y cariño, y dedicación de actividades específicas. La confiabilidad de la escala de igual manera se presenta como favorables respecto a su funcionalidad, lo cual posibilita definir un nivel óptimo de consistencia interna para la adaptación transcultural del instrumento.

En lo que concierne a la estandarización de la escala para la población en la cual fue adaptada, se reconoce la viabilidad de establecer de manera efectiva rangos de puntuación que permitan una clasificación de resultados individuales frente al grupo de referencia (la población de docentes universitarios en el contexto), y su análisis frente a medidas de posicionamiento y medidas de dispersión; esto a través de la baremación propuesta a partir de los resultados obtenidos, con puntos de corte en cuartiles de la distribución de puntajes, en la denominación de niveles "bajo", "moderado", "considerable" y "alto".

Se recomienda, para próximos procesos investigativos, añadir estudios con un tamaño de muestra más considerable lo cual disminuirá el nivel de error estadístico y probablemente mejorará la calidad y la potencia de las propiedades psicométricas de la escala que se proponga. En relación a lo anteriormente mencionado y en pro de optimizar dichas propiedades, también se considera importante obtener más evidencias de validez, distintas a las empleadas en la presente adaptación, lo cual constituirá una cimentación más sólida para las medidas que obtenga el instrumento en procesos aplicados. Además, se ve conveniente emplear población y muestra con diferentes características sociodemográficas, permitiendo un alcance todavía mayor a la posibilidad de emplear la escala, para identificar las características de los Roles de género en diversas circunstancias.

No obstante, tales limitaciones, el presente proceso de validación aporta una herramienta de acercamiento y caracterización de la percepción de sobre los roles de género en contextos universitarios, la cual puede ser usada para identificar cómo se visualizan las dinámicas de roles en docentes universitarios. Esta información puede ser útil tanto en procesos descriptivos como en procesos de intervención, a partir de los cuales se identifiquen las características especificas de las personas con las cuales se trabajará, y con lo cual se pueda aportar a tales procesos en tanto su especificidad y orientación, con el fin de aportar a contextos educativos más incluyentes.

\section{FINANCIACIÓN}

Esta investigación contó con el financiamiento de la convocatoria de investigación docente de la Vicerrectoría de Investigaciones y Proyección Social de la Universidad de Nariño, bajo el marco del proyecto "Calidad de vida laboral y roles de género en docentes universitarios". 


\section{REFERENCIAS BIBLIOGRÁFICAS}

Acker, S. (2003) Géneroyeducación. Reflexiones sociológicas sobre mujeres, enseñanza y feminismo. Madrid, España: Narcea, S.A. de Ediciones.

Acuña, M. (2014) Reflexiones en torno al vínculo género-educación superior. Revista Educación 38(2), 89-10. Recuperado de: https://www.redalyc. org/pdf/440/44031370006.pdf

Anaya, D., y Suaréz, J. (2006) La satisfacción laboral de los profesores en función de la etapa educativa, del género y de la antigüedad profesional, Revista de Investigación Educativa, 24(2), 541556. Recuperado de: https://revistas. um.es/rie/article/view/97181

Andrade, C. (2016) Adaptación y Validación Factorial da Escala de Actitudes Relativas a Roles de Género. Paidéia (Ribeirão Preto). 26(63) 7-14. Recuperado de: https://www.redalyc. org/pdf/3054/305443115001.pdf

Arcos, E., Molina I., Trumper, R., Larrañaga L., del Río, M., Tomic, P., Guerra D., Uarac M., y Szmulewicz, P. (2006) Estudio de perspectiva de género en estudiantes y docentes de la universidad austral de chile. Estudios pedagógicos (Valdivia), 32(2), 27-45. Recuperado de: https://dx.doi.org/10.4067/S071807052006000200002.

Becerra, C. (2015) Calidad de empleo con enfoque de género: propuesta de un marco conceptual. Cuestiones de género: de la igualdad y la diferencia 1 194-224. Recuperado de: https://www.researchgate.net/ publication/279519469_Calidad_de_ empleo con enfoque de genero Propuesta de un marco conceptual
Bisquerra, R. (1989). El análisis multivariante. Barcelona: PPU.

Boff, L. y Muraro, R. (2004) Femenino y masculino. Una nueva conciencia para el encuentro de las diferencias. Madrid: Trotta.

Butler, J. (2001). El género en disputa. El feminismo y la subversión de la Identidad. México: Paidós.

Caballero, M. y Nieto, L. (2015) Nueva gestión pública en Colombia y bienestar laboral del profesorado universitario. Entramado 1 (11) 124-134. Recuperado de: https:// revistas.unilibre.edu.co/index.php/ entramado/article/view/1214

Castillo, M., \& Gamboa, R. (2013) La vinculación de la educación y género. Actualidades Investigativas en Educación, 13(1), 391-407. Recuperado de: https://www. redalyc.org/pdf/447/44725654014.pdf

Castillo, M. y Montes B. (2008) Escala de estereotipos de género actuales. Iniciación a la investigación 2,1-21. Recuperado de: https://www.redalyc. org/pdf/167/16731690027.pdf

Cervantes, V. (2005) Interpretaciones del coeficiente Alpha de Cronbach. Avances en medición, 3. 9-28. Recuperado de: $\quad$ https://www.researchgate. net/publication/259392074 Interpretaciones_del_coeficiente_ alpha_de_Cronbach

Chahín, N. y Briñez, B. (2015) Propiedades psicométricas de la Escala de Ideología de Género en adolescentes colombianos. Universitas psychologica, 14(1) 81-90. Recuperado de: https:// revistas.javeriana.edu.co/index.php/ revPsycho/article/view/1760 
Connell, R. (2001) Educando a los muchachos: nuevas investigaciones sobre masculinidad y estrategias de género para las escuelas. Nómadas (Col) 14, 156-171. Recuperado de: https://www. redalyc.org/pdf/1051/105115268013.pdf

Diaz, R. y Rocha, T. (2005) Cultura de Género, la brecha ideológica entre hombres y mujeres. Anales de Psicología. 21 (1) 42-49 Recuperado de: https://www. um.es/analesps/v21/v21_1/05-21_1.pdf

Eagly, A. (2009) The his and hers of prosocial behavior: an examination of the social psychology of gender. The American Psychologist, 64(8), 644658. Recuperado de: https://doi. org/10.1037/0003-066X.64.8.644

Escobar, J. y Cuervo, A. (2008) Validez de contenido y juicio de expertos: Una aproximación a su utilización. Avances en Medición 6, 27-36 Recuperado de: https://www.researchgate.net/ publication/302438451_Validez_de_ contenido_y_juicio_de_expertos_Una_ aproximacion_a_su_utilizacion.

Escobar, M. (2004) Adaptación transcultural de instrumentos de medida relacionados con la salud. Enfermería Clínica, 14 (2), 102-106 Recuperado de: http:// www.humanas.unal.edu.co/psicometria/ files/7113/8574/5708/Articulo3_Juicio_ de_expertos_27-36.pdf

Fernández, G., Schettin, R., Sánchez, M., Rojo, F., Agulló, M. y João, M. (2018) El papel del género en el buen envejecer. Revista Prisma Social21, 149-176. Recuperado de: https://revistaprismasocial.es/article/ view/2422/0

Fernández-Ballesteros, R. (1996) Introducción a la evaluación Psicológica. España, Madrid: Pirámide.
Ferrando, P. y Anguiano, C. (2010) El análisis factorial como técnica de investigación en psicología. Papeles del Psicólogo, 31(1), 18-33. Recuperado de: https:// www.redalyc.org/pdf/778/77812441003. pdf

Gilbert, D. (1998) Ordinary personology. Nueva York, Estados Unidos: McGraw-Hill.

González, F. y Mora, B. (2014) Características de la violencia de género en la Universidad de Valencia. Escritos de Psicología, 7(2), 36-43 Recuperado de: http://scielo. isciii.es/scielo.php?script=sci_abstract\& pid=S1989-38092014000200005

González, M., Perugache, A., Zambrano, C., Martínez, M., Rodríguez, D., Pantoja, D., Guerrero, A., Zutta, D. y Figueroa, J. (2015) Fortalecimiento de las actitudes tendientes a prevenir las manifestaciones de violencia basada en género en administrativos, docentes y estudiantes en la Universidad de Nariño [Informe]. Pasto: Universidad de Nariño.

Kerlinger, F. y Lee, H. (2002) Investigación del Comportamiento. Métodos de Investigación en Ciencias Sociales. México, McGraw Hill.

Kulis, S., Marsiglia, F. y Nagoshi, J. (2010) Gender Roles, Externalizing Behaviors, and Substance Use Among MexicanAmerican Adolescents. Journal of Social Work Practice in the Addictions, 10(3), 283-307. Recuperado de: https:// www.ncbi.nlm.nih.gov/pmc/articles/ PMC2963473/ 
Ledesma, R., Molina, G. y Valero, P. (2002) Análisis de consistencia interna mediante alfa de Cronbach: un programa basado en gráficos dinámicos. Psico-USF, 7(2). 143-152. Recuperado de: http://pepsic. bvsalud.org/pdf/psicousf/v7n2/v7n2a03. pdf

Lira, M. y Caballero, E. (2020) Adaptación transcultural de instrumentos de evaluación en salud: historia y reflexiones del por qué, cómo y cuándo. Revista Médica Clínica Las Condes, 31(1), 85-94. Recuperado de: https:// www.sciencedirect.com/science/article/ pii/S0716864019301166

López-Molina, S. y Vázquez-Guerrero, M. (2018), "Las políticas de género en una universidadpúblicaestatal:discrepancias institucionales". Revista Iberoamericana de Educación Superior (ries), México, unam-iisue/Universia, 9(25), 138$156 . \quad$ http://dx.doi.org/10.22201/ iisue.20072872e.2019.25.346

Magnusson, D. (1975) Teoría de los test. México, Editorial Trillas.

Maldonado, J. (2015) "No seas puto, camina como se debe" Expresiones de la violencia sexual en estudiantes de Ingeniería. Entramado 2(11) 158-171. Recuperado de: https://revistas.unilibre. edu.co/index.php/entramado/article/ view/1174

Matos, J. (2014) El papel de la figura masculina en la igualdad. Revista IPSE-ds 7, 2136. Recuperado de: http://ipseds.ulpgc. es/IPSE-ds_Vol_7_2014/IPSE-ds\%207. pdf

Montero, I. y León, O. (2007) A guide for naming research studies in Psychology. International Journal of clinical and Health psychology, 7(3),
847-862. Recuperado de: https://www. redalyc.org/pdf/337/33770318.pdf

Morales, P. (2008) Estadística aplicada a las ciencias sociales. Madrid, Universidad Pontificia Comillas.

Muñiz, J. y Fonseca, E. (2019) Diez pasos para la construcción de un test. Psicothema, 31(1). 7-16. Recuperado de: http:// www.psicothema.com/psicothema. asp?id $=4508$

Páramo, P. (2010) Las representaciones de género en profesores universitarios. Estudios pedagógicos (Valdivia), 36(2), 177-193. Recuperado de: https://dx.doi.org/10.4067/S071807052010000200010

Quero, M. (2010) Confiabilidad y coeficiente Alpha de Cronbach. Telos, 21 (2). 248-252. Recuperado de: https://www. redalyc.org/pdf/993/99315569010.pdf

Redlick, M. (2018) Traditional Gender Roles and Their Connections to Relational Uncertainty and Relational Satisfaction. Psychology \& Sexuality, 10(1), 1-16. Recuperado de: https://www.tandfonline. com/doi/abs/10.1080/19419899.2018.1 513422

Román, R., Domínguez, S., Saucedo, M. y Tánori, J. (2016) Validación de un instrumento sobre cultura de género en instituciones de educación, salud e investigación en el Noroeste de México. La ventana. Revista de estudios de género, 5(44), 83-108. Recuperado de: http://www. scielo.org.mx/scielo.php?script=sci_artt ext\&pid=\$1405-94362016000200083

Saldívar, A., Díaz, R., Reyes. N., Armenta, C., López, F., Moreno, M., Romero, A., Hernández, J. y Domínguez, M. (2015) Roles de Género y Diversidad: Validación 
de una Escala en Varios Contextos Culturales. Acta de Investigación Psicológica - Psychological Research Records, 5(3),2124-2148. Recuperado de: $\quad$ https://www.redalyc.org/articulo. oa?id=3589/358943649003

Sales-Oliveira, C., Villas-Boas, S. y Las-Heras, S. (2016), "Estereótipos de género e sexismo em docentes do ensino superior". Revista Iberoamericana de Educación Superior (ries), México, unamiisue/Universia, 7(19), 22-41. https://ries.universia.net/article/ view/1143/estereotipos-generosexismoem-docentes-do-ensino-superior

Samani, S. (2016) Between Texts and Contexts: Contemporary Muslim Gender Roles. Islam and Christian-Muslim Relations 27(3), 319-332. Recuperado de: https:// www.tandfonline.com/doi/abs/10.1080/0 9596410.2016.1148969

Scott, J. (1986) Gender: A Useful Category of Historical Analysis. American Historical Review, 91, 1053-1075. Recuperado de: https://www.jstor.org/ stable/1864376?seq $=1$

Soret, I. (2007). Modelo de medición de conocimiento y generación de ventajas competitivas en el ámbito de la iniciativa "Respuesta eficiente al consumidor", Madrid, España: ESIC Editorial.

Spence, J. y Buckner, C. (2000) Instrumental and expressive traits, trait stereotypes, and sexist attitudes: what do they signify? Psychology of Women Quarterly, 24(1), 44-53. Recuperado de: https:// doi.org/10.1111/j.1471-6402.2000. tb01021.x
Tura, M., Martínez, C., Pons, O. y Simo, P. (2010) Construcción y validación de una escala de medición de igualdad de oportunidades de género. España, XIV Congreso de Ingeniería de Organización.

Valadez, G. y Luna, M. (2017) Validación de una Escala de Mandatos de Género en universitarios de México. Ciencia UAT 12(2): 67-77. Recuperado de: https://www.researchgate.net/ publication/322970095_Validacion_ de_una_Escala_de_Mandatos_de_ Genero_en_universitarios_de_Mexico

Zambrano, C., Perugache, A. y Figueroa, J. (2017) Gender-based violence manifestations in university teachers. Rev. Psicogente, 20(37): 147-160. Recuperado de: http://doi.org/10.17081/ psico.20.37.2424

Zapata, E. y Ayala, M. (2014) Políticas de 252 equidad de género: educación para una escuela libre de violencia. Ra Ximhai: Revista de Sociedad, Cultura y Desarrollo Sustentable, 10(7),1-21. Recuperado de: https://www.redalyc. org/pdf/461/46132451001.pdf 